\title{
Risk for Type 2 Diabetes Among SNAP Participants With Prediabetes
}

\author{
Diana L. Malkin-Washeim \\ Bronx-Lebanon Hospital Center \\ Shirley Gerrior \\ Walden University
}

\begin{abstract}
This study explored food security status among Supplemental Nutrition Assistance Program (SNAP) participants with prediabetes in relation to food choice decisions over a 30-day benefit cycle that potentially increases the risk of Type 2 diabetes. A cross-sectional, quantitative design based on food choice process model constructs was used. SNAP participants $(n=36)$ with prediabetes, aged 21-70 years, were recruited as outpatients from Bronx Lebanon Hospital Center and completed self-reported questionnaires on demographics and health, food security, and food frequency over time. Descriptive statistics, Pearson chi square tests, and regression analysis were performed using SPSS. Two post-hoc tests, the Friedman's test, and Wilcoxon signed-rank test were used to measure the difference in means between food groups. Using a multiple response analysis, the 11 food categories had the greatest variation for Week 1, compared to Weeks 2-4. Use of coping strategies to minimize hunger was limited. Very low food security associated with certain coping strategies disrupted eating patterns that affected food variation over time and increased the intake of non-nutrient-dense foods. Changing SNAP's benefit allotments, and making mandatory, a nutrition package and nutrition education, may increase food security and nutrient-dense food variation thereby decreasing the risk for Type 2 diabetes.
\end{abstract}

Keywords: food security, Type 2 diabetes, coping strategies, nutrition

\section{Introduction}

The Food and Agriculture Organization of the United Nations policy brief from the 1996 World Summit stated that "Food security exists when all people, at all times, have physical and economic access to sufficient, safe and nutritious food that meets their dietary needs and food preferences for an active and healthy life" (Food and Agriculture Organization, 2006, p. 1). The U.S. Department of Agriculture (USDA) defined high food security as "no reported indications of food-access problems or limitations" (2015, para 2). For many lower income households in the USDA's Supplemental Nutrition Assistance Program (SNAP), food security is limited and may be inconsistent over time. The purpose of the present study was to explore the risk of Type 2 diabetes among SNAP participants with prediabetes in regards to their food security status, food choice decisions and coping strategies (when possibly food insecure), over a 30-day benefit cycle.

The theoretical model used for this research was the food choice process model (FCPM; Furst, Connors, Bisogni, Sobal, \& Falk,1996; Sobal \& Bisogni, 2009). In the economic model of food consumption, neighborhood food access was examined, linking access to consumption or health 
outcomes, where the premise is that environments influence behavior. The FCPM has three components: life course, influences, and personal food system. According to Furst et al. (1996), food choice decisions are multifaceted, where life course includes past influences of personal experiences and current involvement, transitions and anticipations of future events; influences are ideals, personal factors, resources, social framework, and food context; and personal food systems are value negotiations (sensory perceptions, monetary considerations, quality, managing relationships, convenience, and health and nutrition). Consequently, food choice decisions are frequent, situational, dynamic, and complex.

According to the USDA (2014), SNAP's monthly benefit allotments add food purchasing power to the incomes of 47.6 million people living in 23.1 million households. However, food security is not attainable in some households, especially in the Northeast, where food prices are higher than those in the Midwest. Among SNAP participants, nearly 50\% are children, $10 \%$ are elderly, and more than $40 \%$ of the participants live in households with an income. According to the Center for the Study of the Presidency and Congress (2012) and the USDA (2013, p. xi), there is no data on the risks for developing Type 2 diabetes based on food choices in relation to food security status among SNAP participants with prediabetes over a 30-day benefit cycle.

For some SNAP households, food security status and poverty are associated with the potential for significant health consequences. Among adults in these households, food choices may be limited, resulting in inadequate dietary intake and their health may be compromised due to postponing medical care (Center for the Study of the Presidency and Congress, 2012). Inadequate dietary intake and postponed medical care increase the risk of obesity and chronic disease (Kushel, Gupta, Gee, \& Haas, 2006). In addition, food insecurity coupled with housing instability leads to barriers to health care among low-income Americans (Kushel et al., 2006). Kushel and colleagues defined housing instability as "as having difficulty paying rent, spending more than $50 \%$ of household income on housing, having frequent moves, living in overcrowded conditions, or doubling up with friends and relatives " (p. 71) and said that " $23.6 \%$ reported housing instability and $42.7 \%$ reported food insecurity" (p. 75) among low-income adults in the United States. Approximately, "70\% of people with prediabetes will develop Type 2 diabetes" (Buysschaert \& Bergman, 2011, pp. 293-294). Hence, by targeting SNAP participants with prediabetes and examining their food choices and coping strategies in relation to food security during the 30 -day benefit cycle, it may offer insights into food choices that increase the risk of developing Type 2 diabetes.

The purpose of the present study is to examine food security status among SNAP participants with prediabetes. The study focuses on food choice decisions from specific food categories as well as coping strategies over a 30-day benefit cycle that potentially increase the risk of Type 2 diabetes. This research is important and relevant because it considers the role of SNAP over the 30-day benefit cycle regarding food choice decisions, food security status, and risk for developing Type 2 diabetes. In addition, there is no known published data on this relationship between food choice decisions, food security and the risk for developing Type 2 diabetes. But given the rise of Type 2 diabetes, the food choices made by SNAP participants with prediabetes are important. According to the New York State Department of Health (2016),

Diabetes has become an epidemic that affects one out of every 10 adult New Yorkers. Since 1994, the number of people in the state who have diabetes has more than doubled, and it is likely that number will double again by the year 2050. (para 2) 
The findings in the present study have the potential to change SNAP's benefit allotments, make nutrition education mandatory, and create a mandatory nutrition food package. This then could reduce food insecure households and reduce the risk of Type 2 diabetes.

\section{Method}

\section{Setting and Participants}

SNAP participants $(n=36)$ were patients at the Bronx-Lebanon Hospital Center (BLHC) and lived in south and central Bronx, New York. The inclusion criteria for the study were as follows: prediabetes as determined by a glycosylated hemoglobin (A1C) of 5.7-6.4\%, literate in English or Spanish, and at least 21 years of age. The exclusion criteria were as follows: diagnosed with gestational diabetes, Type 2, or Type 1 diabetes. Undocumented citizens (foreign-born persons who do not have the right to remain in the United States) and women who were pregnant or breastfeeding were excluded. Candidates were referred through (a) the clinic's primary care physicians, (b) adult endocrinologists, and (c) a registered dietitian at the Health and Wellness Center (an off-campus health clinic of the BLHC and where the data were collected).

\section{Sample}

Between January 2011 and May 2013, 594 patients were diagnosed at BLHC with prediabetes, which was the basis for the sample size. While some of these patients were not available or were not receiving care at the time of the study, this number was used to determine the sample size. The sample size was determined with a confidence interval of 16 , confidence level of $95 \%$, significance level of $p=0.05$, and 50\% effect size (Creative Research Systems, 2012); a one-tail test with a significance of $p=0.05$ and 50\% effect size was used (Creative Research Systems, 2012). The effect size was determined by Cohen's $d=0.50$ and a power of 0.85 ; thus, the sample size of 30 was determined.

\section{Procedure}

There were two Institutional Review Boards, one from the BLHC (\#09-12-13-07) and one from Walden University (\#04-07-14-0161967). A recruitment flyer was distributed to candidates who were then referred to the researcher for screening. A booklet in English and Spanish included a consent form and three self-administered questionnaires: the demographics and health questionnaire; a food frequency questionnaire from the U.S. National Institutes of Health National Cancer Institute (NCI, 2010) called the Dietary Health Questionnaire II (DHQ II); and a food security questionnaire, the Food Security Supplement from the Current Population Survey (CPS-FSS; USDA Economic Research Service, 2014). Participants who completed the three self-administered questionnaires received a $\$ 5.00$ metro card and a $\$ 25.00$ gift card from a local grocery chain.

\section{Research Question}

The research question addressed in this study is as follows:

Research Question: Does the level of food security among SNAP participants with prediabetes, associated with food choice decisions and coping strategies over a 30-day benefit cycle, potentially increase the participants' risk of Type 2 diabetes? 
This research question is built upon the theoretical model, FCPM, to address the what, how, and possibly why certain factors influenced food choice decisions and was designed to capture possible consumption patterns, cycles of eating, and explore coping strategies that might occur as part of influences to food security.

The basis for this research question was to explore the transitory aspect of food security, which overlaps the two components of the FCPM: influences and personal food systems. This question was addressed with the use of the DHQ II and CPS-FSS questionnaires. This research question led to an examination of the FCPM's component, life course, which explores people's food choice trajectories that possibly lead to habitual food decisions that can affect how people adjust to life course transitions.

\section{Design}

This was a cross-sectional, quantitative study with food security as the independent variable. The dependent variables were food choice decisions, coping strategies as determined by food choices, food expenditures, and perceptions of personal health that are influential in food choice decisions as personal health relates to food security status.

To compare food choice variation over time, the 30-day benefit cycle was divided into Week 1 to Week 4. A "control" group was created, using Week 1 from "more" consumption over the 30-day benefit cycle comparing to Weeks 2, 3, and 4 of "more" (experimental group) from the DHQ II that reflect 11 food groups: sweetened beverages; dairy; meat (beef, hot dogs, bacon); cold cuts; chicken, pork, fish, and ground beef; fast foods; starchy vegetables; fruits; bread, rolls, rice, spaghetti, and macaroni; desserts; and nonstarchy vegetables. The modification to the research design by creating a control and experimental group improved the ability to assess the impact of the food security status (independent variable). For this additional analysis, the Friedman's test and the Wilcoxon signedrank test were used. The Friedman test checked for differences between dependent variables/groups, which are measured at the ordinal level (Laerd Statistics, 2013). The "more" weeks were comparedWeek 1 to Week 2, Week 1 to Week 3, Week 1 to Week 4, Week 2 to Week 3, Week 2 to Week 4, and Week 3 to Week 4-to determine differences between groups over the 30-day food cycle for the 11 food categories using Weeks 1 through 4 as data points.

\section{Survey Tools}

There were three self-administered questionnaires used to collect data: demographics and health questionnaire, a food frequency questionnaire, and a food security questionnaire. The demographics and health questionnaire was developed to collect social demographics: socioeconomics, age, weight and height (to determine body mass index [BMI]), perception of health, ethnicity, race, and education level. These data were used to explore an association between perception of health and BMI, food security and BMI, and demographics of the sample. The DHQ II, an NCI (2010) food frequency questionnaire with 194 questions, targeted specific foods and macronutrients (fat and carbohydrates), beverages, fruits, and vegetables. It was modified for three reasons: to eliminate portion sizes, to include foods that reflect the cultures sampled, and to add specific questions on food frequency, and thus food variation, over the 4 weeks of a 30-day benefit cycle. Food frequency was assessed for 11 foods groups: (a) sweetened beverages; (b) dairy; (c) meat (beef, hotdogs, bacon); (d) chicken, pork, fish, and ground beef; (e) cold cuts; (f) fast foods (at certain restaurants); (g) starchy 
vegetables; (h) fruits; (i) breads, rice, rolls, spaghetti, and macaroni; (j) desserts; and (k) nonstarchy vegetables.

To explore variation of food choices, the 30-day benefit cycle was divided into 4 weeks (Week 1 to Week 4), and a control and experimental group were created. The control group was created using Week 1 from "more" consumption over the 30-day benefit cycle. The control group was then used to compare to Weeks 2, 3, and 4 of "more" (experimental group) from the DHQ II that reflected the 11 food groups assessed using the food frequency questionnaire.

The food security questionnaire used was the 30-day reference food security questionnaire (the FSS) and validated by the USDA (2014). The FSS questionnaire included 50 items and was used to explore how much money was spent for food, types of food programs that supplemented food consumption, how long food lasts, and coping strategies used when there is not enough food over the 30-day benefit cycle. Certain sections of the FSS tool were used along with the 10-item U.S. Adult Food Security Module, which is a subset to the FSS tool. This allowed for the exploration of different locations where food is purchased, actual versus usual money spent on food, money spent on nonfood items, minimum spending needs to have enough food, coping strategies, and supplemental food program participation.

The FSS was used to explore food security status and food choice decisions, measured with the DHQ II based on times consumed for high fat and sugary beverages. The NCI's (2010) DHQ II and the CPS-FSS U.S. Adult Food Security Module (USDA Economic Research Service, 2014) both are federally sponsored, established, verified, and validated instruments.

Food security at high and marginal levels and food insecurity (defined as low food security and very low food security status; USDA, 2016) were explored in relation to food choice decisions. Food security status was calculated based on the number of affirmative responses to the 10-item FSSM questionnaire (USDA, 2000, p. 34).

\section{Methodology: Food Security}

Food security status was calculated in relation to coping strategies and the two additional subquestions to the 10-item questionnaire, U.S. Adult Food Security Module (USDA Economic Research Service, 2012) were included to reflect the frequency of how often participants, over the last 30 days ate less because there was not enough money for food and how often the participant experienced hunger because there was not enough money for food. Hence, there were 12 questions used for coding the food security status raw scores, which was assigned the following codes: $0=$ high food security, 1-2 = marginal food security, 3-5 = low food security, and 6-12= very low food security. Any item not filled in was considered negative versus missing. The responses of yes, often true, sometimes true, almost every month, and some months but not every month were coded as an affirmative response.

\section{Statistical Analysis}

SPSS Version 21 was used to code the three self-administered questionnaires. Descriptive statistics was used for the demographics and health questionnaire and food security status. Regression analysis was used to measure an association among food security, food choice decisions, coping strategies, and perception of personal health status. A Pearson chi-square test was used to measure categorical variables (gender, food choice decisions, education, use of food assistance programs, high 
fat foods, sugary beverages, and frequency of food consumption over a 30-day benefit cycle). Two post hoc tests, the Friedman's test, a nonparametric test, and the Wilcoxon signed-rank test were used to measure the difference in means between food groups.

\section{Results}

Of the study's 36 participants, one lived in a shelter and was waiting to receive his SNAP benefits; however, he was allowed to participate in the study. As shown in Table 1, 66.7\% reported being Hispanic/Latino, $36.1 \%$ lived alone, $52.8 \%$ did not graduate from high school, only $25 \%$ had attended some college, and $83.4 \%$ perceived their health as fair to poor.

The BMI $\left(\mathrm{kg} \times 703 / \mathrm{ht}^{2}\right)$ was manually calculated: $5.6 \%$ were of normal weight $\left(18.50-24.99 \mathrm{~kg} / \mathrm{m}^{2}\right)$, $19.4 \%$ were overweight $\left(25.0-29.99 \mathrm{~kg} . \mathrm{m}^{2}\right), 22.2 \%$ were Obese Class I $\left(30.0-34.99 \mathrm{~kg} / \mathrm{m}^{2}\right), 13.9 \%$ were Obese Class II (35-39.99 kg/m²), and $22.2 \%$ were Extremely Obese Class III ( $\geq 40 \mathrm{~kg} / \mathrm{m}^{2}$; see Table 1). A relationship between perception of health status and BMI was not statistically significant $(p>.05$, $r=0.059)$.

Of the participants in this study, $94.59 \%$ were very low food secure and $5.60 \%$ were low food secure; none were marginal or high food secure. When food security status and BMI were examined, $21.42 \%$ through $28.57 \%$ participants were very low food secure and were either overweight, Obese Class I, Obese Class II, or Extremely Obese Class III, respectively (see Table 2).

In exploring coping strategies in regards to food running out over time (see Table 3), 52.8\% responded "true" that they were worried that food would run out, food they bought would not last, and they had no money to buy more food; $44.4 \%$ responded "true" that they could not afford to eat balanced meals.

In exploring coping strategies in response to food budgeting over time (see Table 4), $55.6 \%$ cut the size of or skipped meals, $22.2 \%$ stretched food or money, $66.1 \%$ ate less, $25 \%$ did not eat for a whole day, and $25 \%$ received emergency food; however, $13.9 \%$ received meals at a soup kitchen, and $11.5 \%$ either had meals delivered to the home or went to a community program.

In examining coping strategies over time (see Table 5), 36.1\% ate less because there was not enough money for food, $30.6 \%$ participants cut the size of or skipped meals, $13.9 \%$ were hungry but did not eat, and $8.3 \%$ did not eat for a whole day because there was not enough money for food almost every month. 
Table 1: Sociodemographic Characteristics at Bronx-Lebanon Hospital Center

\begin{tabular}{|c|c|c|c|c|c|}
\hline Characteristics & $N$ & $\%$ & Characteristics & $N$ & $\%$ \\
\hline Age (y) & & & Living situation & & \\
\hline$<21$ & 0 & 0 & Alone & 13 & 36.1 \\
\hline $22-30$ & 2 & 2.6 & With wife & 2 & 5.6 \\
\hline $31-40$ & 4 & 11.1 & With husband & 9 & 25.0 \\
\hline $41-50$ & 11 & 30.6 & With children & 10 & 27.8 \\
\hline $51-60$ & 11 & 30.6 & With friend & 5 & 13.9 \\
\hline $61-70$ & 8 & 22.2 & Adult weight status & & \\
\hline Gender & & & $\begin{array}{l}\text { Normal (18.50-24.99 } \\
\left.\mathrm{kg} / \mathrm{m}^{2}\right)\end{array}$ & 2 & 5.6 \\
\hline Female & 26 & 72.7 & $\begin{array}{l}\text { Overweight (25.0-29.99 } \\
\left.\mathrm{kg} / \mathrm{m}^{2}\right)\end{array}$ & 7 & 19.4 \\
\hline Male & 10 & 27.8 & $\begin{array}{l}\text { Obese Class I (30-34.99 } \\
\left.\mathrm{kg} / \mathrm{m}^{2}\right)\end{array}$ & 8 & 22.2 \\
\hline Perception of health & & & $\begin{array}{l}\text { Obese Class II (35.0-39.99 } \\
\left.\mathrm{kg} / \mathrm{m}^{2}\right)\end{array}$ & 5 & 13.9 \\
\hline Excellent & 1 & 2.9 & $\begin{array}{l}\text { Extreme Obesity Class III } \\
\left(>40 \mathrm{~kg} / \mathrm{m}^{2}\right)\end{array}$ & 8 & 22.2 \\
\hline Good & 4 & 11.1 & Missing & 6 & 16.7 \\
\hline Fair & 15 & 41.7 & $\begin{array}{l}\text { Last grade of schooling } \\
\text { completed }\end{array}$ & & \\
\hline Poor & 15 & 41.7 & $1-6$ & 7 & 19.4 \\
\hline Missing & 1 & 2.8 & $7-9$ & 11 & 30.6 \\
\hline Country of origin & & & $10-12$ & 17 & 47.2 \\
\hline United States & 11 & 30.6 & Graduated high school & & \\
\hline Puerto Rico & 5 & 13.9 & Yes & 16 & 44.4 \\
\hline Dominican Republic & 11 & 30.6 & No & 19 & 52.8 \\
\hline Haiti & 2 & 5.6 & Missing & 1 & 2.8 \\
\hline Mexico & 2 & 5.6 & Attended some college & & \\
\hline Other & 4 & 11.1 & Yes & 9 & 25.0 \\
\hline Missing & 1 & 2.8 & No & 26 & 72.2 \\
\hline Ethnicity & & & Missing & 1 & 2.8 \\
\hline Hispanic/Latino & 24 & 66.7 & Graduated college & & \\
\hline Non-Hispanic/non-Latino & 3 & 8.3 & Yes & 5 & 13.9 \\
\hline Black American & 7 & 19.4 & No & 29 & 80.6 \\
\hline Missing & 2 & 5.6 & Missing & 1 & 2.8 \\
\hline
\end{tabular}

Note. One participant lived in a shelter and was in-between in receiving Supplemental Nutrition Assistance Program benefits but participated in the research study. 
Table 2: Food Security Status and Body Mass Index (BMI)

\begin{tabular}{|c|c|c|c|c|}
\hline \multirow{3}{*}{$\begin{array}{l}\text { Weight } \\
\text { Classification } \\
\text { BMI }\left(\mathrm{kg} / \mathrm{m}^{2}\right)\end{array}$} & \multicolumn{4}{|c|}{ Food Security Status } \\
\hline & \multicolumn{2}{|c|}{ Low Food Security } & \multicolumn{2}{|c|}{ Very Low Food Security } \\
\hline & $N$ & $\%$ & $N$ & $\%$ \\
\hline $\begin{array}{l}\text { Normal (18.50- } \\
24.99)\end{array}$ & 0 & 0 & 2 & 7 \\
\hline $\begin{array}{l}\text { Overweight } \\
(>25.00)\end{array}$ & 1 & 50 & 6 & 21.42 \\
\hline $\begin{array}{l}\text { Obese Class I } \\
(30.00-34.99)\end{array}$ & 1 & 50 & 7 & 25 \\
\hline $\begin{array}{l}\text { Obese Class II } \\
(35.00-39.99)\end{array}$ & 0 & 0 & 5 & 17.85 \\
\hline $\begin{array}{l}\text { Extreme } \\
\text { Obesity Class } \\
\text { III }(>40.00)\end{array}$ & 0 & 0 & 8 & 28.57 \\
\hline Total & 2 & & 28 & \\
\hline
\end{tabular}

Note. Low food security = reporting of reduced quality, variety, or desirability of diet, and little or no indication of reduced food intake; very low food security = reporting of multiple indications of disrupted eating patterns and reduced food intake (USDA Economic Research Service, 2012).

Table 3: Responses to Coping Strategies: Food Ran Out, Food Did Not Last, and Could Not Afford Balanced Meals

\begin{tabular}{|c|c|c|c|c|c|c|c|c|}
\hline \multirow[b]{3}{*}{ Coping Strategies } & \multirow{2}{*}{\multicolumn{2}{|c|}{ Often True }} & \multicolumn{2}{|c|}{ Sometimes } & \multicolumn{2}{|c|}{ Never } & \multirow{2}{*}{\multicolumn{2}{|c|}{ Missing }} \\
\hline & & & \multicolumn{2}{|c|}{ True } & \multicolumn{2}{|c|}{ True } & & \\
\hline & $N$ & $\%$ & $N$ & $\%$ & $N$ & $\%$ & $N$ & $\%$ \\
\hline Worried food would run out & 12 & 33.3 & 19 & 52.8 & 5 & 13.9 & & \\
\hline $\begin{array}{l}\text { Food bought did not last and no } \\
\text { money to buy more food }\end{array}$ & 9 & 25.0 & 19 & 52.8 & 7 & 19.4 & 1 & 2.8 \\
\hline $\begin{array}{l}\text { Could not afford to eat balanced } \\
\text { meals }\end{array}$ & 12 & 33.3 & 16 & 44.4 & 7 & 19.4 & 1 & 2.8 \\
\hline
\end{tabular}


Table 4: Coping Strategies: In Response to Food Budgeting Over a 30-day Benefit Cycle

\begin{tabular}{lcccc}
\hline & \multicolumn{2}{c}{ Yes } & \multicolumn{2}{c}{ No } \\
\cline { 2 - 5 } Coping Strategies & $N$ & $\%$ & $N$ & $\%$ \\
\hline Cut size or skipped meals (SH2) & 20 & 55.6 & 14 & 38.9 \\
Stretched food or money (S8E) & 8 & 22.2 & 24 & 66.7 \\
Ate less (SH3) & 22 & 66.1 & 12 & 33.3 \\
Did not eat for a whole day (SSH1) & 9 & 25.0 & 22 & 61.1 \\
Received meals delivered to the home & 4 & 11.15 & 31 & 86.1 \\
(SC1) & & & & \\
Went to a community program (SC2) & 4 & 11.15 & 32 & 88.9 \\
Received emergency food (SC3) & 9 & 25.0 & 27 & 75.0 \\
Received meals at soup kitchen or shelter & 5 & 13.9 & 31 & 86.1 \\
(SC4)
\end{tabular}
(SC4)

Note. Coping strategies from the Current Population Survey: Food Security Supplement (SH2, S8E, SH3, $\mathrm{SSH} 1, \mathrm{SC} 1, \mathrm{SC} 2, \mathrm{SC} 3, \mathrm{SC} 4)$ : meals delivered to home from community programs; emergency food from church, a food pantry, or food bank.

Table 5: Coping Strategies: In Response to Food Budgeting Over a 30-Day Benefit Cycle

\begin{tabular}{|c|c|c|c|c|c|c|c|c|c|c|}
\hline \multirow[b]{2}{*}{ Coping Strategies } & \multicolumn{2}{|c|}{$\begin{array}{l}\text { Almost } \\
\text { Every } \\
\text { Month }\end{array}$} & \multicolumn{2}{|c|}{$\begin{array}{l}\text { Some Months } \\
\text { But Not } \\
\text { Every Month }\end{array}$} & \multicolumn{2}{|c|}{$\begin{array}{l}\text { Only } 1 \text { or } 2 \\
\text { Months }\end{array}$} & \multicolumn{2}{|c|}{ Skippeda } & \multicolumn{2}{|c|}{ Missing } \\
\hline & $N$ & $\%$ & $N$ & $\%$ & $N$ & $\%$ & $N$ & $\%$ & $N$ & $\%$ \\
\hline $\begin{array}{l}\text { Cut the size of or } \\
\text { skipped meals }\end{array}$ & 11 & 30.6 & 10 & 27.8 & 1 & 2.8 & 14 & 38.9 & & \\
\hline $\begin{array}{l}\text { Ate less because there } \\
\text { was not enough money } \\
\text { for food }\end{array}$ & 13 & 36.1 & 7 & 19.4 & 3 & 8.3 & 12 & 33.3 & 1 & 2.8 \\
\hline $\begin{array}{l}\text { Was hungry but did not } \\
\text { eat because not enough } \\
\text { money for food }\end{array}$ & 5 & 13.9 & 9 & 25.0 & 3 & 8.3 & 19 & 52.8 & & \\
\hline $\begin{array}{l}\text { Did not eat for a whole } \\
\text { day because not enough } \\
\text { money for food }\end{array}$ & 3 & 8.3 & 8 & 22.2 & 1 & 2.8 & 22 & 61.1 & 2 & 5.6 \\
\hline
\end{tabular}

a Participants were instructed to skip this if responding "no" to the question, "In the last 30 days, did you or other adults in your household ever not eat for a whole day because there wasn't enough money for food?" from the Current Population Survey: Food Security Supplement questionnaire. 


\section{Food Choice Decisions}

Based on the Friedman's test, there was a statistically significant difference in food consumption based on which week of the month was compared, $\chi^{2}(2)=23.480, p=.009$, between related groups. However, because it was not known exactly where those differences lay (11 food categories from DHQ II "more" only), a Wilcoxon signed-rank test was run as a post hoc test to explore where the differences actually occurred with a Bonferroni correction (applied), using a significant level of $p<$ .005. Prior to the Wilcoxon signed-rank test, the researcher calculated the Bonferroni adjustment $(.05 / 10[\#$ of total tests] $=.005$, hence the new significance value $)$.

Results from the Friedman and Wilcoxon tests comparing "more" (Week 1 and Week 4) were significant between all food groups $(p<.005)$. The only food category that showed nonsignificant results was sweetened beverages. The 11 food categories were analyzed using a multiple response analysis. The results were 105 (54.1\%) responses that were "more" during Week 1 in relation to the 2nd, 3rd and 4th weeks (27.3\%, 10.3\%, and 8.2\%, respectively). Specifically exploring food categories in the control group, "more" Week 1, with the exception of desserts at $13.0 \%$, all of the other food categories ranged from $19 \%$ to $30.6 \%$ compared to Weeks 2 , 3, and 4 , which ranged from $2.8 \%$ to $19.4 \%$. In terms of the frequency of the food categories, "more" (which supplemented the Friedman and Wilcoxon analyses) showed that there were more varied food choices during Week 1 compared to Weeks, 2, 3, and 4 over the 30-day benefit cycle. There were no significant differences for the food choices between Weeks 2 and 3, between Weeks 2 and 4, or between Weeks 3 and 4.

One component of the FCPM, personal systems construct, is food choice values that classify foods and situations according to peoples' values (Sobal, Bisogni, Devine, \& Jastran, 2006, p. 7). The six most noted negotiable values are "sensory perceptions, monetary considerations, convenience, health/nutrition, managing relationships and quality" (Furst et al., 1996, p. 257). The sample participants perceived their health as fair to poor, and it is possible that because almost two fifths ran out of SNAP benefits by Week 3 (see Table 6), they are economically distressed, and further, it is possible that because of this, their perception of health is altered. Of the sample, $37.14 \%$ were living on $\$ 3.33-\$ 6.40$ per day and $22.85 \%$ were living on $\$ 6.66-\$ 11.66$ per day, calculated by how many dollars per day a participant may be living on based on SNAP benefits per month (see Table 7).

Table 6: Supplemental Nutrition Assistant Program (SNAP) Benefit Allotments

\begin{tabular}{lcccc}
\hline & & \multicolumn{2}{c}{$\begin{array}{c}\text { SNAP Benefits to Run Out } \\
\text { Over the 30-Day Benefit Cycle }\end{array}$} \\
\cline { 4 - 5 } Month & $N$ & $\%$ & $N$ & $\%$ \\
\hline 1st & 14 & 38.9 & 2 & 5.6 \\
2nd & 15 & 41.7 & 9 & 25 \\
3rd & 4 & 11.1 & 14 & 38.9 \\
4th & 1 & 2.8 & 8 & 22.2 \\
Missing & 2 & 5.6 & 3 & 8.3 \\
Total & 36 & & 36 & \\
\hline
\end{tabular}


Table 7: Supplemental Nutrition Assistant Program Benefits per Month

\begin{tabular}{lccc}
\hline $\begin{array}{l}\text { Dollars per } \\
\text { Month }\end{array}$ & $N$ & $\%$ & Dollar Average per Day per Householda \\
\hline$\$ 0-\$ 199$ & 13 & 37.14 & $\$ 3.33-\$ 6.40$ (i.e., $\$ 100-\$ 192 /$ month) \\
$\$ 200-\$ 399$ & 8 & 22.85 & $\$ 6.66-\$ 11.66$ (i.e., $\$ 200-\$ 350 /$ month) \\
$\$ 400-\$ 499$ & 3 & 8.57 & $\$ 13.33-\$ 16.00$ (i.e., $\$ 400-\$ 480 /$ month) \\
$\$ 500-\$ 700$ & 0 & 0 & $\$ 0 /$ month \\
$\$ 730$ & 1 & 2.8 & $\$ 24.33$ (i.e., $\$ 730 /$ month) \\
Missing & 1 & 2.8 & \\
Total & 36 & & \\
\hline
\end{tabular}

a The number of people in the household is not available to calculate how many dollars per person per day.

The two factors, running out of SNAP benefits during Weeks 3 and 4 of the 30-day benefit cycle and living on $\$ 3.00-\$ 6.00$ per day, may contribute to non-nutrient-dense food choices (saturated fats and sweetened sugary beverages). Consequently, Malik, Popkin, Bray, Despres, and Hu (2011) argued that SNAP participants with prediabetes who make these food choices are at an increased risk for obesity, insulin resistance, and Type 2 diabetes. With prediabetes, food choices of saturated fats and sweetened beverages, and limited financial resources at a specific point in time as seen in this study, the participants' personal perception of fair-to-poor health supports the personal food systems construct of the FCPM as negotiating values such as sensory perceptions, monetary considerations, relationship management, convenience, and health and nutrition. Hence, food choice decisions are situational and complex.

\section{Significant Findings}

The sample participants were either overweight or extremely obese with very low food security status. The sample participants also reported a change of food choices over a 30-day benefit cycle with more variation within 11 food categories in Week 1 than in Weeks 2, 3, and 4 and that their health was perceived fair to poor.

The eight coping strategies explored to help minimize food insecurity and hunger (cutting the size of or skipping meals, eating less, not eating for a whole day, receiving emergency food, stretching food or money, receiving meals delivered to the home, participating in a community program, and eating meals at a soup kitchen or shelter; see Tables 4 and 5) were found to be limited over the 30-day benefit cycle. Food security status was found to influence coping strategies; however, food security was not a good predictor of specific coping strategies. The strength of the relationship (between food security status and coping strategies) was significant $(r=.597, p<.01)$. In addition, there was no statistically significant association for perception of health and BMI.

Coping strategies were dominated by stretching money for food, cutting food into smaller pieces or skipping meals, or eating less because there was no money for food. With no statistically significant difference between the level of food security and the eight coping strategies, and with food security not being a good predictor of specific coping strategies, it appears that food security does not affect coping strategies over a 30-day benefit cycle. 
There were significant differences with food choices between all of the food categories for "more" Week 1 and "more" Week 3 and between all of the food categories between "more" Week 1 and "more" Week 4. However, there was no difference between Weeks 2 and 3, between Weeks 2 and 4, and between Weeks 3 and 4.

SNAP participants who have prediabetes appear to make different food choice decisions between the beginning of the 30-day food cycle and the later part of the 30-day food cycle. Hence, food security in relation to food choices and coping strategies appears to change over the 30-day benefit cycle among SNAP participants who have prediabetes and potentially increases their risk for Type 2 diabetes.

\section{Discussion}

The study's findings are relevant to the increased risk for Type 2 diabetes. The USDA Economic Research Service (2015) defined several ranges of food security: High food security reflects no limitations to food access, whereas marginal food security reflects one or two reported indications of food access along with anxiety over food sufficiency or shortage of food in the house and no indication of changes in food intake. On the other extreme, low food security refers to reports of reduced quality, variety, or desirability of diet, and there is little or no indication of reduced food intake, while very low food security reflects multiple indications of disrupted eating patterns and there is reduced food intake.

The analysis of the food security data from this study showed an insufficient amount of food available, in that SNAP participants consumed more foods during Week 1 compared to Weeks 2, 3, and 4 over a 30-day benefit cycle. In addition, reported intake for most foods was inconsistent from week to week with the exception of the bread, rice, rolls, spaghetti, macaroni and nonstarchy vegetables categories. In addition, participants did not use some of the resources that would supplement food intake, as seen by the limited use of food pantries, soup kitchens, community programs, and meals being delivered to the home; hence, food access was limited. This may be due to "timing" as to when food insecurity exists and when there is the need to access food and nutrition assistance.

The concept of cyclical eating is a pattern of eating for a specific period of time and then, due to a restriction of food based on limited resources, there is very limited food consumption. This has been known to increase body fat with overeating. Cyclical food restriction is associated with an increase of body fat, a decrease of lean muscle mass, and a quicker weight regain where the body is responding to refeeding (Dinour, Bergen \& Yeh, 2007). This is an example of the feast-famine cycle that has been linked to SNAP (Dinour, et al., 2007). Cyclical eating is referred to as a reference period of 3 weeks of overeating followed by 1 week of involuntary food restriction due to the depletion of food sources; then the cycle resumes once the benefits from SNAP resume (Dinour et al., 2007). The increase of adipose tissue and decrease of lean muscle mass contributes to insulin resistance, a risk factor for developing Type 2 diabetes. The present research data collected captured variation of foods "more" during Weeks 1 and 4 not "more" between Weeks 2 and 3, not between Weeks 2 and 4, and not between Weeks 3 and 4. With SNAP benefits in general running out between Weeks 3 and 4 (see Table 6), the data reflects a possible pattern of cyclical eating.

Most of the participants in the present study were very low food secure and either overweight or extremely obese (Class III). In addition, more than a third of the participants lived alone. Therefore, it is plausible that weight may fluctuate depending on eating relationships with others and tastes, 
both justifying certain food choice decisions that influence long-term health conditions over someone's lifetime.

Wardle and colleagues (2004) argued the female "survival advantage" (p. 107) is a relatively modern phenomenon and has varied over time and among different countries. Thus, this has added significance to health behavior differences among genders. Wardle et al. (2004) argued that males may eat fewer fresh fruits and vegetables, choose fewer high-fiber foods, eat few lower calorie foods, and consume more soft drinks than do women, and women's consumption tends to be higher in micronutrients than that of men. In addition, men's food choices are rated less important than those of women. Wardle et al. (2004) explored food choice behaviors among 23 countries and concluded that gender differences in food choices appear to be attributed to women's greater weight control involvement and a strong belief in healthy eating. However, the authors did not address culture as a social determinant in relation to food choice decisions. The present study did not explore gender in relation to food choice decisions, although among the demographics, there were more females $(72.7 \%)$ than males (27.8\%) who participated in the study. However, the data did explore ethnicity. The sample was predominately Hispanic/Latino and culture may have played a significant role in food choice decisions as reflected in the data collected with the DHQ II exploring the customary approach to self-identity.

There are various coping strategies used to overcome economic barriers, such as federal or emergency food assistance programs; however, the data indicate many of these resources were underused. The participants reported feelings of hunger and economic distress equivalent to being very low food secure, as well as having limited education.

Ideals, personal experiences, resources, social factors, and food contexts are five factors that influence and shape food choice decisions. Each of these factors may fluctuate over a lifetime when making food choices (Furst et al., 1996). A social determinant, such as culture, that includes family, peers, and meal patterns plays a key role in self-identity, hence influencing food choice decisions. In addition, there are certain barriers that affect food choice decisions, such as cost, transportation, cooking skills, or limited resources that may also influence food security status. Food security fluctuates over time and strategies to cope facilitate various food choice decisions, which affect the short- and long-term health outcome. The participants' coping strategies to minimize food insecurity and hunger were limited in the 30-day benefit cycle. Very low food security that was associated with limited coping strategies disrupted eating patterns and possibly affected food variation over time with an increase of non-nutrient-dense foods, thus increasing the risk of obesity and Type 2 diabetes. The timing of SNAP benefit allotments, food security status, education, language, culture, financial resources, and perception of health all influence food choices and affect health outcome.

Culture is one of the most pervasive foundations for food choices, and SNAP participants in the Bronx are culturally diverse. According to the New York State Department of Health (2014) the population in the Bronx has multiple health and socioeconomic challenges and is considered the least healthy county in New York State, along with high rates of chronic disease states including obesity and diabetes. The collected data align with the New York State Department of Health's (2014) report, noting that there is insecurity with respect to housing, unsafe environment, and poor access to healthy food.

Although many cultures sustain their cultural food identity when coming to a new country, there is also food acculturation as people integrate. As people from varied societies enter the food system of 
the West, there is a nutrition transition or dietary shift to consume more energy-dense fats, saturated fats, and sugars, moving away from coarse grains and starchy roots (Nestle et al., 1998). Over two thirds of the subjects in this study were of Hispanic/Latino origin, and food acculturation and urbanization from rural living may play a role in nutrition transition for these participants where availability of processed foods increases energy-dense-type foods (sugar, salt, and fat). Specific ethnic foods were reported in the study a minimum of twice per week, reflecting a customary approach to self-identity, such as rice, green bananas, cassava with onions, dried beans, yogurt, tostones, and fried plantains. According to Bisogni, Connors, Devine, and Sobal (2002), there are selfidentities in relation to food consumption such as, eating practices, and personal characteristics in referencing groups and social categories.

To date, there is no universal tool to measure food security. However, the USDA (2014) food security questionnaire was used in the present study to measure this phenomenon. Barrett (2010) argued that survey tools can target coping strategies index, food expenditures, and dietary diversity measures responses to past consumptions. However, using aggregate food availability as an indicator is not a good predictor of food insecurity, because the undernourished population has increased by $9 \%$ globally, in relation to a $12 \%$ rise in global food production per capita since 1990 . In addition, food insecurity is seasonal or irregular, depending on unemployment, health status, and other adverse events, such as surgical procedures and chronic conditions such as disability and/or drug abuse.

Food security is strengthened when there are policies in place to reduce poverty, increase access to healthy foods, and implement safety nets for vulnerable households such as SNAP participants. If tools for food security measure a diagnosis, then researchers need to look at what will measure the greatest response to food insecurity. Authorizing a mandatory SNAP nutrition package that includes fresh, frozen, or canned with no added salt, no added sugar, fresh fruits and vegetables, whole grain cereals, and healthy snacks, as well as a mandatory nutrition education component, may increase quality nutrition and decrease the risk for developing Type 2 diabetes.

As of May 20, 2016, the U.S. Food and Drug Administration (2016) announced a new nutrition fact label that would include "added sugars," in grams and as a percent daily value. This additional label data could make nutrition education in regards to sugar content easier to understand-how much sugar is actually in a given product and how much one is actually consuming. The proposed mandatory nutrition education would include label reading and coping strategies to help increase quality nutrition, reduce barriers to running out of food, and possibly make food last over the 30-day benefit cycle. It is also proposed that the education would include the benefits of shopping more frequently for fresh or frozen fruits, vegetables, and other nutrient-dense foods that have protein; calcium; vitamins A, C, and D; and fiber. Healthier outcomes among SNAP participants with prediabetes are feasible when fresh or frozen produce is included in food choices.

Implementing an optional food allotment plan per month would benefit SNAP participants and increase food availability over the 30-day benefit cycle. In addition, it may offset the expense of produce throughout the month for households in SNAP to purchase produce in season, eliminating the transportation costs built into the produce that is out of season, and to purchase frozen vegetables as a way to increase the vegetables' longevity. The optional benefit allotment may decrease the risk of food insecurity, cyclical weight gain, missed meals at the end of the month, and consumption of high fat and non-nutrient-dense foods; increase intake of produce; and improve overall quality nutrition, thereby helping to decrease the risk for developing Type 2 diabetes. 
The ability to be food secure is a fundamental human right, as it is to eliminate the emotional stress among SNAP participants who find themselves using specific coping strategies to decrease hunger and to make food last until the next benefit allotment. It would be a significant public health achievement to be able to distribute the benefits over the 30-day benefit cycle to a choice of once or twice over the same time period, create mandatory nutrition education, and a mandatory food package for those households participating in SNAP. This would greatly decrease the status of low to very low food security that influences choices of non-nutrient-dense foods, hence lowering the risk for obesity and for developing Type 2 diabetes. In addition, as seen in this study, food choice variation is affected by SNAP benefit allotments, food security status, and coping strategies.

Since the present study was conducted, the USDA (2016) has announced that SNAP participants will have increased access to healthy foods by requiring retail stores that accept SNAP to stock a wider array of food choices. According to the USDA, there are over 260,000 current retailers nationwide that are authorized to redeem SNAP benefits, and these retailers would be required to offer seven varieties of qualifying foods for sale on a continuous basis, along with perishable foods. The staple food groups are dairy, breads and cereals, meats, poultry and fish, and fruits and vegetables (USDA, 2016). This is a good attempt to address availability of quality foods among those with limited incomes, challenging transportation, language barriers, and food and language illiteracy. The challenge may still exist in accessing the retail stores that carry these food items in areas that tend to be limited to fast food establishments. It would be prudent of the USDA to provide all SNAP participants the name and locations of retailers in their particular area that redeem SNAP benefits.

\section{Limitations}

Because this was a cross-sectional study, causation cannot be inferred. The sample size was small and limited by sample characteristics, such that study results should not be generalized beyond the group under study and the sample $(n=36)$. These limitations may restrict the power of the study to show whether the results are a real effect or, by chance, in regards to outcome. With the small sample size, a further limitation is the assumption that the data has a normal distribution and homogeneity of the variance; hence, it is more challenging to achieve normality, and the $t$-test results could be misleading.

The completion of the self-administered questionnaires was challenging for some participants due to the language or words used, the length of the survey tools, and the time it took to complete the questionnaires; hence, the responses may not be completely accurate. The frequency questionnaires used to determine food-related behaviors and social dynamics might not truly reflect actual events but rather usual events over the 30-day benefit cycle. The DHQ II questions that reflected the 11 food groups were problematic for coding due to multiple responses making the responses potentially unreliable or inaccurate. In exploring the coping strategies to determine a transition time when coping strategies were (mostly) used, an exact point in time over the 30-day benefit cycle could not be determined, as well as if there was a transition of time from marginal to low food security or from low food security to very low food security status.

\section{Recommendations}

Having a larger sample would increase the validity of the variables under study, capture a larger frequency of foods, and provide a better understanding of food choice decisions. To increase insight into the health behaviors or provide a better understanding of barriers to food choice decisions, a 
qualitative research study would be needed. Examining similar variables over a longer period of time, such as 6-12 months, may increase the reliability and validity of the data used to determine food choice variation over time. This longer period of time would more clearly identify the "transition" period of food security status; whether there are differences in access of food assistance programs; and whether coping strategies are more, less, or the same. In addition, the longer timeframe would help pinpoint when food choice decision differences have occurred. To avoid respondent fatigue and confusing questions, shortening the DHQ II would help the participants' responses. To add extra insights into food choice decisions, modifications are recommended to the CPS-FSS questionnaire to explore cooking skills, access to a kitchen, and access to various kitchen tools that affect types of foods purchased, preparation, and consumption.

SNAP participants who have prediabetes are at risk for developing Type 2 diabetes, especially if they find themselves using specific coping strategies to decrease hunger. These coping strategies influence food choice variation over time and the potential consumption of non-nutrient-dense foods. However, with mandatory nutrition education and food packaging for those households participating in SNAP, this would greatly decrease the low to very low food security status that influences non-nutrientdense food choices, hence lowering the risk for obesity and Type 2 diabetes.

\section{References}

Barrett, C. B. (2010). Measuring food insecurity. Science, 327, 825-828. Retrieved from http://www.sciencemag.org/content/327/5967/825.shor

Bisogni, C. A., Connors, M., Devine, C. M., \& Sobal, J. (2002). Who we are and how we eat: A qualitative study of identities in food choice. Journal of Nutrition Education and Behavior, 34, 128-139. doi:10.1016/S1499-4046(06)60082-1

Buysschaert, M., \& Bergman, M. (2011). Definition of prediabetes. Medical Clinics of North America, 95, 289-297. doi:10.1016/j.mcna.2010.11.002

Center for the Study of the Presidency and Congress. (2012). SNAP to health: A fresh approach to improving nutrition in the supplemental nutrition assistance program. Retrieved from http://www.snaptohealth.org/wp-content/uploads/2012/10/CSPC-SNAP-Report.pdf

Creative Research Systems. (2012). Sample size calculator. Retrieved from http://www.surveysystem.com/sscalc.htm

Dinour, L. M., Bergen, D., \& Yeh, M.-C. (2007). The food insecurity-obesity paradox: A review of the literature and the role of food stamps may play. Journal of the American Dietetic Association, 107, 1952-1961. doi:10.1016/j.jada.2007.08.006

Food and Agriculture Organization of the United Nations. (2006). Policy brief: Food security. Retrieved from http://www.fao.org/forestry/13128-0e6f36f27e0091055bec28ebe830f46b3.pdf

Furst, T., Connors, M., Bisogni, C. A., Sobal, J., \& Falk, L. W. (1996). Food choice: A conceptual model of the process. Appetite, 26, 247-266. doi:10.1006/appe.1996.0019

Kushel, M. B., Gupta, R., Gee, L., \& Haas, J. S. (2006). Housing instability and food insecurity as barriers to health care among low-income Americans. Journal of General Internal Medicine, 21, 71-77. doi:10.1111/j.1525-1497.2005.00278.x

Laerd Statistics. (2013). Friedman test in SPSS. Retrieved from https://statistics.laerd.com/spsstutorials/friedman-test-using-spss-statistics.php 
Malik, V. S., Popkin, B. M., Bray, G. A., Despres, J. P., \& Hu, F. B. (2011). Sugar sweetened beverages, obesity, Type 2 diabetes and cardiovascular disease risk. Circulation, 121, 13561364. doi:10.1161/circulationAHA.109.876185

National Institutes of Health National Cancer Institute (NCI). (2010). Risk factor monitoring and methods: Validation studies for the Diet History Questionnaire II. Retrieved from http://riskfactor.cancer.gov/dhq2/about/validation.html

Nestle, M., Wing, R., Birch, L., DiSogra, L., Drewnowski, A., Middleton, S., \& Economos, C. (1998). Behavioral and social influences on food choice. Nutrition Reviews, 56, S50-S64. doi:10.1111/j.1753-4887.1998.tb01732.x

New York State Department of Health. (2014). New York City Health Provider Partnership: Bronx community needs assessment: Final draft November 14, 2014. Retrieved from http://www.health.ny.gov/health_care/medicaid/redesign/dsrip/pps_applications/docs/st_barn abas_hosp_dba_sbh_health_system/3.4_st_barnabas_cna.pdf

New York State Department of Health. (2016). Diabetes. Retrieved from http://www.health.ny.gov/diseases/conditions/diabetes/

Sobal, J., \& Bisogni, C. A. (2009). Constructing food choice decisions. Annals of Behavioral Medicine, 38, S37-S46. doi:10.1007/s12160-009-9124-5

Sobal, J., Bisogni, C. A., Devine, C. M., \& Jastran, M. (2006). A conceptual model of the food choice process over the life course. In R. Shepard \& M. Raats (Eds.), The psychology of food choice (pp. 19-39). Cambridge, MA: CABI.

U.S. Department of Agriculture (USDA). (2000). Guide to measuring household food security. Retrieved from http://www.fns.usda.gov/sites/default/files/FSGuide_0.pdf

U.S. Department of Agriculture (USDA). (2013). SNAP food security in-depth interview study. Retrieved from http://www.fns.usda.gov/snap-food-security-depth-interview-study

U.S. Department of Agriculture (USDA). (2016). USDA proposes policies to improve food access, healthy choices for low-income Americans. Retrieved from http://www.usda.gov/wps/portal/usda/usdahome?contentid=2016/02/0045.xml\&contentidonl

U.S. Department of Agriculture (USDA) Economic Research Service. (2012): CNSTAT assessment/ food insecurity and hunger in the United States: An assessment of the measure. Retrieved from http://www.ers.usda.gov/topics/food-nutrition-assistance/food-security-in-theus/definitions-of-food-security/cnstat-assessment.aspx\#.UXh0p4Krnbx

U.S. Department of Agriculture (USDA) Economic Research Service. (2014). Survey tools: U.S. household food security survey module. Retrieved from http://www.ers.usda.gov/topics/foodnutrition-assistance/food-security-in-the-us/survey-tools.aspx

U.S. Department of Agriculture (USDA) Economic Research Service. (2015). Definitions of food security. Retrieved from http://www.ers.usda.gov/topics/food-nutrition-assistance/foodsecurity-in-the-us/definitions-of-food-security.aspx

U.S. Food and Drug Administration (2016). Changes to the nutrition facts label. Retrieved from http://www.fda.gov/Food/GuidanceRegulation/GuidanceDocumentsRegulatoryInformation/La belingNutrition/ucm385663.htm\#highlights 
Wardle, J., Haase, A. M., Steptoe, A., Nillapun, M., Jonwutiwes, K., \& Bellisie, F. (2004). Gender differences in food choice: The contribution of health beliefs and dieting. Annals of Behavioral Medicine, 27, 107-116.

The Journal of Social, Behavioral, and Health Sciences is an open-access, peer-reviewed, online interdisciplinary journal focusing on research findings that address contemporary national and international issues. Its objectives are to (a) encourage dialogue between scholars and practitioners in the social, behavioral, and health sciences that fosters the integration of research with practice; (b) promote innovative models of interdisciplinary collaboration among the social, behavioral, and health sciences that address complex social problems; and (c) inform the relationship between practice and research in the social, behavioral, and health sciences.

Walden University Publishing: http://www.publishing.waldenu.edu 